\title{
Cloning and Expression of Major Surface Antigen 1 Gene of Toxoplasma gondii RH Strain Using the Expression Vector pVAX1 in Chinese Hamster Ovary Cells
}

\author{
Rahman Abdizadeh $^{1,2}$; Sharif Maraghi ${ }^{2,3}$; Ata A. Ghadiri ${ }^{4, *}$; Mehdi Tavalla ${ }^{2}$; Saeedeh Shojaee ${ }^{5}$ \\ ${ }^{1}$ Cell and Molecular Research Center, School of Medicine, Ahvaz Jundishapur University of Medical Sciences, Ahvaz, IR Iran \\ ${ }_{3}^{2}$ Department of Medical Parasitology, School of Medicine, Ahvaz Jundishapur University of Medical Sciences, Ahvaz, IR Iran \\ ${ }^{3}$ Institute of Health Research, Thalassemia and Hemoglobinopathy Research Center, Ahvaz Jundishapur University of Medical Sciences, Ahvaz, IR Iran \\ ${ }_{5}^{4}$ Department of Immunology, School of Medicine, Ahvaz Jundishapur University of Medical Sciences, Ahvaz, IR Iran \\ ${ }^{5}$ Department of Medical Parasitology and Mycology, School of Public Health, Tehran University of Medical Sciences Tehran, IR Iran \\ ${ }^{*}$ Corresponding author: Ata A. Ghadiri, Department of Immunology, School of Medicine, Ahvaz Jundishapur University of Medical Sciences, Ahvaz, IR Iran. Tel: +98-6133330144, Fax: \\ +98-6133330145, E-mail: ata.ghadiri@hotmail.fr
}

Received: August 5, 2014; Revised: September 30, 2014; Accepted: October 29, 2014

\begin{abstract}
Background: Toxoplasmosis is an opportunistic protozoan infection with a high prevalence in a broad range of hosts infecting up to onethird of the world human population. Toxoplasmosis leads to serious medical problems in immunocompromised individuals and fetuses and also induces abortion and mortality in domestic animals. Therefore, there is a huge demand for the development of an effective vaccine. Surface Antigen 1(SAG1) is one of the important immunodominant surface antigens of Toxoplasma gondii, which interacts with host cells and primarily involved in adhesion, invasion and stimulation of host immune response. Surface antigen 1 is considered as the leading candidate for development of an effective vaccine against toxoplasmosis.

Objectives: The purpose of this study was to clone the major surface antigen1 gene (SAG1) from the genotype 1 of T. gondii, RH strain into the eukaryotic expression vector PVAX1 in order to use for a DNA vaccine.

Materials and Methods: Genomic DNA was extracted from tachyzoite of the parasite using the QIAamp DNA mini kit. After designing the specific primers, SAG1 gene was amplified by Polymerase Chain Reaction (PCR). The purified PCR products were then cloned into a pPrime plasmid vector. The aforementioned product was subcloned into the pVAX1 eukaryotic expression vector. The recombinant pVAX1-SAG1 was then transfected into Chinese Hamster Ovary (CHO) cells and expression of SAG1 antigen was evaluated using Reverse Transcriptase Polymerase Chain Reaction (RT-PCR), Immunofluorescence Assay (IFA) and Western Blotting (WB).

Results: The cloning and subcloning products (pPrime-SAG1 and pVAX1-SAG1 plasmid vectors) of SAG1 gene were verified and confirmed by enzyme digestion and sequencing. A $30 \mathrm{kDa}$ recombinant protein was expressed in CHO cells as shown by IFA and WB methods.

Conclusions: The pVAX1 expression vector and CHO cells are a suitable system for high-level recombinant protein production for SAG1 gene from T. gondii parasites and are promising approaches for antigen preparation in vaccine development.
\end{abstract}

Keywords: Cloning; Toxoplasma gondii; SAG1 antigen; CHO Cell

\section{Background}

Toxoplasma gondii is a widespread, obligate and intracellular protozoan parasite belonging to the phylum Apicomplexa that infects any nucleated cell of a wide range of vertebrate animals, including mammalian and avian species and causes the most common zoonotic infection in humans $(1,2)$. The only known definitive hosts for this organism are cats and other felines while all nonfeline vertebrates including humans, domestic and wild livestock, birds and marine mammals act as intermediate hosts of this protozoan $(3,4)$. Humans become infected postnatal horizontally by ingestion or handling of undercooked or raw meat containing viable tissue cysts, consuming food (fruits and vegetables) and water contaminated with sporulated oocysts or drinking of unpasteurized milk of infected animals.

Humans could be infected accidentally by ingesting oocysts from the environment with exposure to contaminated soil (gardening without gloves) or cat litter, organ transplantation or blood transfusion from infected donors and laboratory accidents. Another way of infection is by tachyzoites that are passed to the fetus via the placenta when an uninfected mother acquires infection during pregnancy (5-8). Toxoplasmosis was estimated to infect about one-third of world's human population, but its prevalence is affected by the strain of parasite (and genotype), age, geographical situation, occupational groups, food habits, social culture and thus varies from less than $10 \%-90 \%$ in different countries $(9,10)$.

Toxoplasmosis in healthy adult humans is usually asymptomatic chronic form or associated with self-limited symptoms; therefore, often goes unnoticed and rarely needs treatment (11). Meanwhile, immunocompromised

Copyright (C) 2015, Ahvaz Jundishapur University of Medical Sciences. This is an open-access article distributed under the terms of the Creative Commons Attribution-NonCommercial 4.0 International License (http://creativecommons.org/licenses/by-nc/4.0/) which permits copy and redistribute the material just in noncommercial usages, provided the original work is properly cited. 
patients and pregnant women who acquire their infection during gestation, fetuses and newborns who are congenitally infected and those with chorioretinitis are the serious examples of toxoplasmosis. However, immunocompromised individuals, such as patients with AIDS and persons undergoing therapies for malignancies, transplants or lymphoproliferative disorders because of reactivation of a latent infection may present severe forms of the disease, such as encephalitis and pneumonitis $(12,13)$. Another risk group consists of children infected during pregnancy, depending on the gestational age at which the pregnant woman acquires the infection, the consequences may vary from death of the fetus in utero and spontaneous abortion to hydrocephalus, microcephaly, retinochoroiditis in the surviving infants $(14,15)$. Moreover, the parasite causes economic losses to the livestock industry mainly among food producing animals as the infection may lead to embryonic death, abortion, mummification, still birth and neonatal loss in pregnant animals especially sheep, goats and pigs, as well as a source of transmission to humans (16). Thus, toxoplasmosis continues to be a real problem in public health and livestock husbandry.

The primary strategy for the treatment of toxoplasmosis is chemical drugs; however, they are poorly tolerated, have side effects, drug-resistance and are not an effective treatment for tissue cysts of $T$. gondii in humans and animals also they cannot prevent reinfection (17). Therefore, the development of an effective and safe vaccine against $T$. gondii infection is an important, urgent goal and appears to be achievable. Some antigens of $T$. gondii are relatively effective candidates for DNA vaccine includes Surface Antigen Glycoproteins (SAGs), Excretory-Secretory Dense Granule Proteins (GRAs), Rhoptry Proteins (ROPs), and Micronemal Proteins (MICs), which were tested in recent years (17-19). Surface antigen 1 (SAG1) is one of the SAGs of T. gondii that anchors to the cell membrane of this protozoan by a glycosylphosphatidylinositol anchor and plays an important role in the processes of host cell attachment, penetration and host immune evasion (20-22).

Although this protein only accounts for $3 \%-5 \%$ of total proteins of this parasite it is the most abundant SAGs in the tachyzoites of T. gondii and during toxoplasmosis, the majority of the antibodies are reactive against it. Surface antigen 1 is a hydrophobic $30 \mathrm{kDa}$ protein with an acidic $\mathrm{pH}$ that has been identified in the parasitophorous vacuole membrane and in the intravacuolar membranous network. The gene of SAG1 has a single-copy with a length of $1.1 \mathrm{~kb}$ that does not have introns in the coding region or a TATA box in the promoter region $(19,23)$. In the present study, we cloned and expressed TgSAG1 from RH strain of $T$. gondii and analyzed its gene expression in Chinese Hamster Ovary (CHO) cells.

\section{Objectives}

The purpose of this study was to clone SAG1 gene from the genotype 1 of T. gondii, RH strain in eukaryotic expression vector pVAX1. Moreover, the major objective of the present study was to express SAG1 using the eukaryotic expression vector pVAX1 in eukaryotic cells to evaluate the high-level recombinant protein production use for DNA vaccine in the future.

\section{Materials and Methods}

The polymerase chain reaction (PCR) cloning Kit was purchased from 5prime (Germany) and other kits were purchased from Qiagen (Germany), restriction endonucleases were purchased from New England Biolabs (New England Biolabs Inc., USA). T4 DNA ligase, Taq polymerase, dNTP and protein weight markers were purchased from Roche (Germany). 5-Bromo-4-chloro3-indolyl $\beta$-D-galactopyranoside (X-gal) and Isopropyl$\beta$-D-thiogalactopyranoside (IPTG) were purchased from Sigma-Aldrich Corporation (Germany). The Escherichia coli strain DH5 $\alpha$ and pVAX1 expression vector were purchased from Novagen (Novagen Inc., Madison, WI, USA). All chemicals were obtained from Merck (Germany), Sigma-Aldrich (Germany) and HiMedia (India) Corporations.

\subsection{Parasite}

The tachyzoites of the T. gondii RH strain were inoculated in peritoneal cavity of BALB/c mice. After 3 - 4 days, the parasites were collected, washed and suspended in Phosphate Buffer Saline (PBS, pH 7.2) for lysate preparation and DNA extraction (24).

\subsection{Gene Amplification of Surface Antigen 1}

Genomic DNA of T. gondii RH strain was extracted using the QIAamp DNA Mini Kit (Qiagen, Hilden, Germany) according to the manufacturer's instructions. A pair of primers based on the full protein-coding region of the T. gondii SAG1 gene sequence (Accession number GQ253075) was designed by the DNASIS version 2.6 primer designer software with NheI and XhoI restriction sites at $5^{\prime}$ end of forward and reverse primers, respectively. The sequences were SAG1F: $5^{\prime}$-AAGCTAGCCACCATGTCGGTTTCGCTGCACC-3' and SAG1R: 5' -AGGAATTCTCGAGCTCACGCGACACAAGCTGC-3' (Bioneer Corporation, South Korea). The simple Kozak translational consensus sequence was introduced in the beginning of the PCR product by its addition to the forward primer. Also, the PCR reaction was performed in a total volume of $50 \mu \mathrm{L}$ using 100 ng DNA, $1 \mu \mathrm{L}$ forward and reverse primers at 25 pmol, $50 \mathrm{~m} \mathrm{M} \mathrm{Mgcl}_{2}, 200 \mu \mathrm{M} \mathrm{d} \mathrm{NTP,10X} \mathrm{PCR} \mathrm{buffer,} \mathrm{and}$ $2.5 \mathrm{u}$ Taq polymerase (high fidelity, Roche, Mannheim, Germany).

The PCR condition was as follows: predenaturation at $94^{\circ} \mathrm{C}$ for 5 minutes; denaturation at $94^{\circ} \mathrm{C}$ for 1 minute, annealing at $60^{\circ} \mathrm{C}$ for 30 seconds and extension at $72^{\circ} \mathrm{C}$ for 70 seconds, followed by 30 cycles; final extension at 
$72^{\circ} \mathrm{C}$ for 10 minutes in a thermal cycler (Bio-Rad, Hercules, CA, US). The PCR product was analyzed by electrophoresis on $1 \%$ agarose gel in $1 \mathrm{X}$ TBE buffer and visualized using the ethidium bromide staining on UV transilluminator. The PCR product was purified from the agarose gel using QIAquick gel extraction kit (Qiagen, Hilden, Germany) according to the manufacturer's recommendation and DNA concentration was measured by spectrometry at OD260 and OD280 using Nanodrop 2000 (Thermo Scientific, USA).

\subsection{Cloning of SAG1 Gene}

The PCR product was cloned into the pPrime vector via $\mathrm{T} /$ A cloning method using the 5 prime perfect PCR cloning Kit (5prime, Hamburg, Germany) according to the manufacturer's instructions. Then the pPrime-SAG1 was transformed in calcium chloride E. coli DH5 $\alpha$ competent cells (Novagen Inc., Madison, Wis) and dispensed on Luria-Bertani (LB) medium (HiMedia, Mumbai, India) agar plates containing kanamycin (50 mg/L). Bacterial colonies were screened by agar plate containing Xgal (Sigma, USA) and IPTG (Sigma, USA) to discriminate between recombinant (white) and no recombinant (blue) plasmid containing ones (25). Several suspected colonies (white) were further analyzed by colony PCR. After selecting recombinant clones, the plasmid DNA was extracted from cells cultured overnight with shaking at $250 \mathrm{rpm}$ at $37^{\circ} \mathrm{C}$ by using the Miniprep plasmid purification kit (Qiagen, Hilden, Germany) following the manufacturer's instructions. The selected recombinant plasmids were verified by restriction digestion with NheI and XhoI restriction enzymes (New England BioLabs, Ipswich, MA, USA) and sequenced using M13 primers.

\subsection{Subcloning of Surface Antigen 1 Gene}

The pPrime vector carrying the SAG1 gene and the eukaryotic expression vector pVAX1 (Invitrogen, Carlsbad, USA) were digested by NdeI and XhoI restriction enzymes. After thermal inactivation of the restriction enzymes and analysis by LMP agarose gel electrophoresis, the linearized pVAX1 plasmid and the SAG1 gene were extracted from the \%1 agarose gel by agarose gel DNA extraction kit (Qiagen, Hilden, Germany). The purified linear vector and insert were subjected to ligation reaction using T4 DNA ligase (Roche, Mannheim, Germany). E. coli DH5 $\alpha$ competent cells were transformed with $2 \mathrm{uL}$ of ligation product. Transformed E. coli cells were selected on LB medium agar plates containing kanamycin (50 mg/L). Several colonies were assayed by colony PCR using specific primers (SAG1F and SAG1R). After selecting recombinant clones, the plasmid DNA was extracted from the cells cultured overnight by using the Miniprep plasmid isolation kit (Qiagen, Hilden, Germany) and confirmed by PCR, restrictionenzyme digestion, followed by DNA sequencing using $\mathrm{T} 7$ and BGH primers.

\subsection{Expression of pVAX-Surface Antigen 1 Plasmid in Chinese Hamster Ovary CHO cells}

\subsubsection{Transient Transfection}

Chinese Hamster Ovary (CHO) cell line, were maintained in our laboratory and routinely cultured in Dulbecco's Modified Eagle's Medium (DMEM) supplemented with penicillin $(100 \mathrm{IU} / \mathrm{mL})$, streptomycin $(100 \mathrm{mg} / \mathrm{mL})$ and $10 \%$ Fetal Bovine Serum (FBS) at $37^{\circ} \mathrm{C}$ with $5 \% \mathrm{CO}_{2}$ atmosphere. One-day before transfection, $1-3 \times 10^{4} \mathrm{CHO}$ cells were seeded in 6-well plates (35-mm wells). The recombinant pVAX1-SAG1 plasmid was transfected into cells with X-tremeGENE9 DNA transfection reagent (Roche) according to the manufacturer's instructions. In this way, $3 \mu \mathrm{L}$ X-tremeGENE9 DNA transfection reagent and 100 $\mu \mathrm{L}$ serum-free medium were mixed and incubated for 5 minutes at room temperature and $1 \mu \mathrm{g}$ DNA was added to the mixture and incubated 20 minutes after it was added to a 6-well plate at 70\% - 90\% confluency and plates were incubated for additional 48 hours at $37^{\circ} \mathrm{C}$ in $5 \% \mathrm{CO}_{2}, 95 \%$ humidity. Chinese hamster ovary transfected with pVAX1 without insert and un-transfected cells were used as controls. Then, SAG1 gene expression in transfected cells was evaluated by RT-PCR, immunofluorescence assay and western blot analysis.

\subsubsection{Reverse Transcriptase Polymerase Chain Reaction Assay (RT-PCR)}

The transfected cells and the control cells were rinsed with ice-cold PBS for three times and RNA was extracted using the Trizol reagent (Invitrogen) according to the manufacturer's instructions. DNase I digestion was also performed to minimize the possibility of genomic DNA contamination. The extracted total RNA $(1 \mu \mathrm{g})$ was employed for first-strand cDNA synthesis using $20 \mu \mathrm{L}$ of AccuPower RT-Premix (Bioneer, Korea) at $55^{\circ} \mathrm{C}$ for 1 hour and $95^{\circ} \mathrm{C}$ for 5 minutes. cDNA from the reverse transcription reaction was then used in the subsequent PCR reactions with SAG1F and SAG1R primers.

\subsubsection{Immunofluorescence Assay}

The SAG1 expression in the cells was detected using an indirect immunofluorescence assay (IFA). In brief, the cells were fixed with cooled-acetone, and washed with PBS-0.1\% Triton-X-100 (PBS-T) for three times. The cells were incubated with 1:100 diluted mouse anti-T. gondii p30 monoclonal antibody (Abcam, USA) diluted at room temperature for 60 minutes and washed three times by PBS-T, followed by incubation in 1:300 diluted FITC-labeled goat antimouse IgG antibody (Santa Cruz Biotechnology, Inc., USA) at room temperature for 60 minutes. After washing three times with PBS-T, incubation was performed in dark with DAPI (4', 6-Diamidino-2-phenylindole) at RT for 5 minutes and monolayers were covered with glyc- 
erine and examined for specific fluorescence under an Olympus IX70 multi-parameter fluorescence microscope (Olympus, Tokyo, Japan) (26). Photographs were taken at the magnification $\times 100$ using a canon camera.

\subsubsection{SDS-PAGE Sodium Dodecyl Sulfate-Poly-acryl- amide Gel Electrophoresis and Western Blot}

The transfected and untransfected cells were washed with ice-cold PBS and collected on ice with $200 \mu \mathrm{L}$ RIPA lysis buffer (50 mM Tris pH 7.4, $150 \mathrm{mM} \mathrm{NaCl}, 1 \%$ Triton $\mathrm{X}-100,1 \%$ Sodium deoxycholate, $0.1 \%$ SDS) containing protease inhibitor cocktail (p2714, Sigma, USA). The cells were then exposed to freeze-thaw cycles. Then the cell lysates were boiled in the loading buffer for 10 minutes and separated using a 12\% (w/v) Sodium dodecyl Sulfate-Poly-acrylamide Gel Electrophoresis (SDS-PAGE) according to the standard protocol (27). Following electrophoresis, separated proteins were transferred to Poly-vinylidene Fluoride (PVDF) membrane (Roche Diagnostics GmbH) using a Mini trans-blot electrophoretic transfer cell (Bio-Rad, Hercules, CA, USA) in transfer buffer containing $25 \mathrm{mM}$ Tris ( $\mathrm{pH}=8.3), 192 \mathrm{mM}$ glycine and $20 \%$ methanol at $55 \mathrm{v}$ for 1 hour at $4^{\circ} \mathrm{C}(28)$. The membrane was blocked with $5 \%$ $(\mathrm{w} / \mathrm{v})$ skimmed milk diluted in Tris-buffered saline with Tween 20 (PBS-T) (0.5 M NaCl, $0.02 \mathrm{M}$ Tris pH $=8.5,0.05 \%$ Tween 20) for 1 hour at room temperature. The membrane was furthermore incubated with the mouse antiSAG1 antibody (dilution 1:50) at $37^{\circ} \mathrm{C}$ for 3 hour, followed by three times washing in TBS-T. Then the membrane was incubated with diluted goat antimouse IgG Horseradish Peroxidase (HRP)-labeled secondary antibody (1:10,000; Abcam, USA) for $1 \mathrm{~h}$. The blot was then washed three times with TBS-T and visualized with ECL (Enhanced Chemiluminescence reagents, Amersham, UK) by CCD camera (Bio-Rad, USA).

\section{Results}

\subsection{Cloning and Sequencing of Surface Antigen 1}

Genomic DNA of Toxoplasma tachyzoites was extracted from infected mouse peritoneum and its SAG1 gene was amplified using specific primers, showing a band about 1020 bp on agarose gel (Figure 1). The purified PCR product (1020 bp fragment) was first cloned into a pPrime vector and then subcloned into a pVaxi. Following, transformation and plating of the bacterial cells on LB agar containing the selective antibiotic, IPTG and XGal, the presence of white and blue colonies on LB agar plates containing IPTG and X-Gal was an indication of successful transformation. Several transformed colonies were analyzed by colony PCR with the SAG1 gene specific primers. The selected plasmids (called pPrime-SAG1) were verified by restriction digestion. Sequence analysis of the isolated SAG1 gene with reported genes from and plasmid also confirmed that there were no amplification
Figure 1. Agarose Gel Electrophoresis of the Result of the Amplified SAG1 Gene by PCR

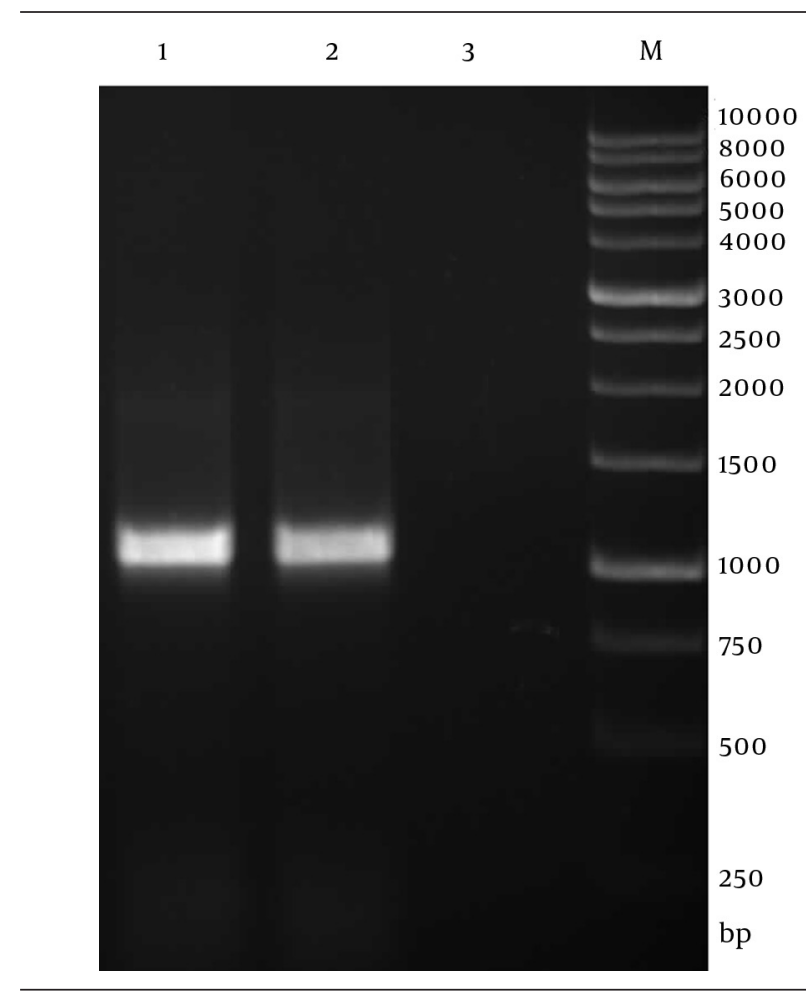

Line 1, 2, 1020bp PCR product; Line 3, negative control; Line M, 1kb DNA ladder.

Figure 2. Agarose Gel Electrophoresis Analysis of Purified pVAX-SAG1 Recombinant Plasmids From Five Colonies on 1\% Agarose Gel Line.

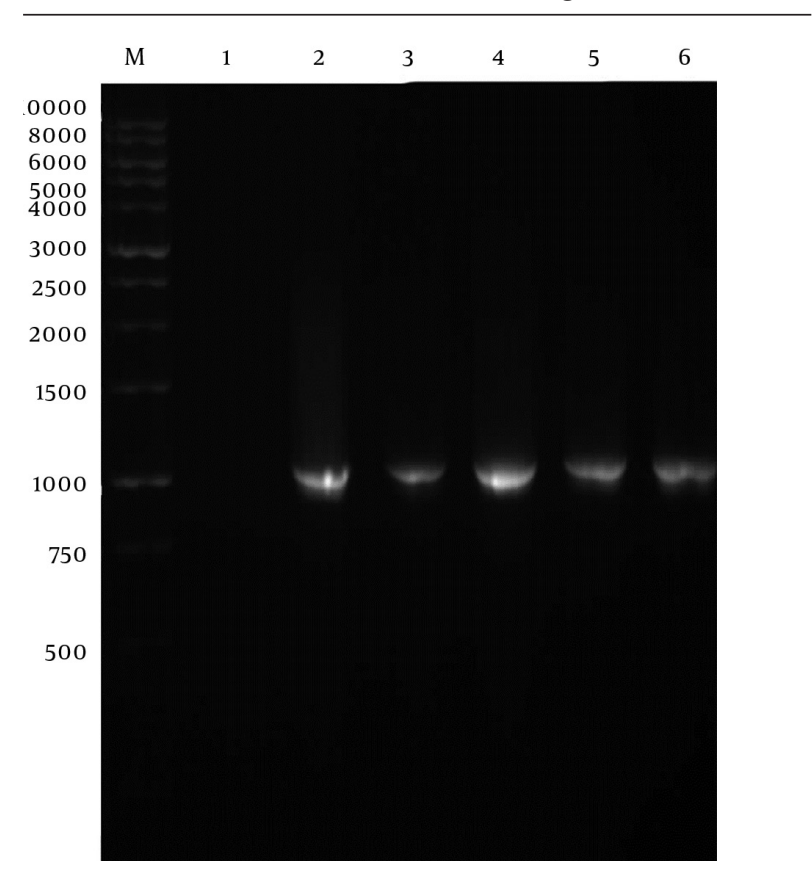

M, 1 kb DNA ladder; Lane 1, negative control; Lane 2 - 6: selected pVAX1-SAG1 recombinant plasmids. 
errors in the cloned SAG1 gene sequence. The sequencing result was also confirmed through comparing against databases and applying Basic Local Alignment Search Tool (Blast) software. After the confirmation of a recombinant pPrime-SAG1 plasmid, the recombinant plasmid was isolated and subcloned into a pVAX1 plasmid. After transformation of E. coli $\mathrm{DH} 5 \alpha$, recombinant plasmids were analyzed using the colony PCR and PCR on the isolated plasmids (Figure 2), restriction digestion (Figure 3 and 4) and sequencing (Figure 5).

\subsection{Expression of pVAX1-Surface Antigene 1 Plas- mid in Chinese Hamster Ovary Cells}

In vitro expression of the pVAX1-SAG1 was evaluated by RT-PCR, IFA and western blot at 48 hours posttransfection. Reverse Transcriptase Polymerase Chain Reaction Assay (RT-PCR) was used to determine the expression of the SAG1 gene in transduced CHO cell line; therefore, the amplified PCR fragment of 1020 bp on transfected cells was electrophoresed on agarose gel and visualized by the ethidium bromide staining that indicated the expression of the SAG1 in eukaryotic cells (Figure 6). As shown in Figure 7, the specific green fluorescence was observed in CHO cells transfected with pVAX1-SAG1, indicated expression of SAG1 protein located in cytoplasm, but not in the negative control cells transfected with the same amount of empty pVAX1 (Figure 7). Further analysis of CHO cell lysates transfected with pVAX1-SAG1 and empty pVAX1 on 12\% SDS-PAGE and western blotting showed that the $\mathrm{CHO}$ cells were efficiently transfected and recombinant SAG1 was detected as a $30 \mathrm{kDa}$ band equivalent to the native protein. In contrast, no immunoreactive material was found in the lysate of untransfected CHO cells and transfected cells with empty pVAX1 plasmid (Figure 8).

\section{Discussion}

Toxoplasmosis is one of the most important infectious diseases in immunocompromised human and nonimmune pregnant woman $(12,15)$. In veterinary, it causes tremendous problems for livestock husbandry including abortion and stillbirth in pregnant animals. Therefore, prompt diagnosis, effective treatment and prevention of this parasite is necessary to minimize the harm of this infection in humans and animals (23). Because of diagnostic problems and ineffective therapeutic approaches, vaccine development might be one of the important ways for prevention of toxoplasmosis. Thus, many studies investigated immune-prophylaxis by different immunogenic components of T. gondii $(18,19)$. Among them, DNA vaccination is a novel strategy in immunization field which encoding antigenic proteins of pathogens by plasmid DNAs that induced efficiency humoral and cellular immunities against a wide spectrum of infectious and noninfectious agents (29).

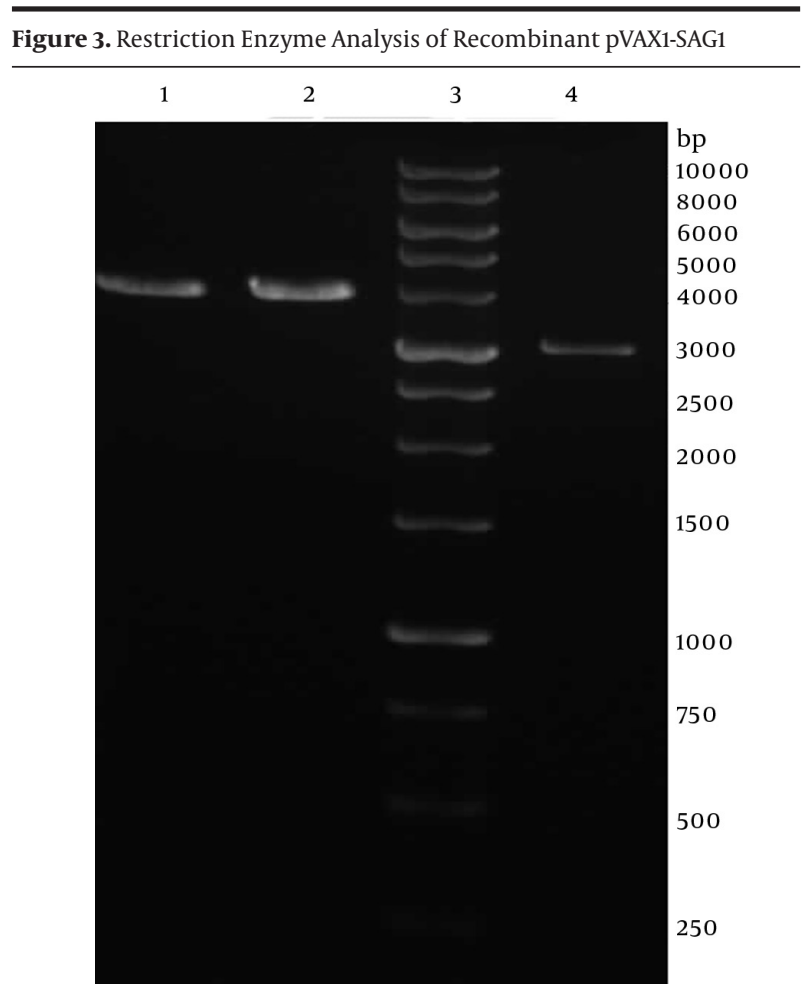

Line 1 and 2, the recombinant PVAX-SAG1 plasmid digested by NheI; Line 3, $1 \mathrm{~kb}$ DNA ladder; Line 4, the pVAX vector digested by NheI.

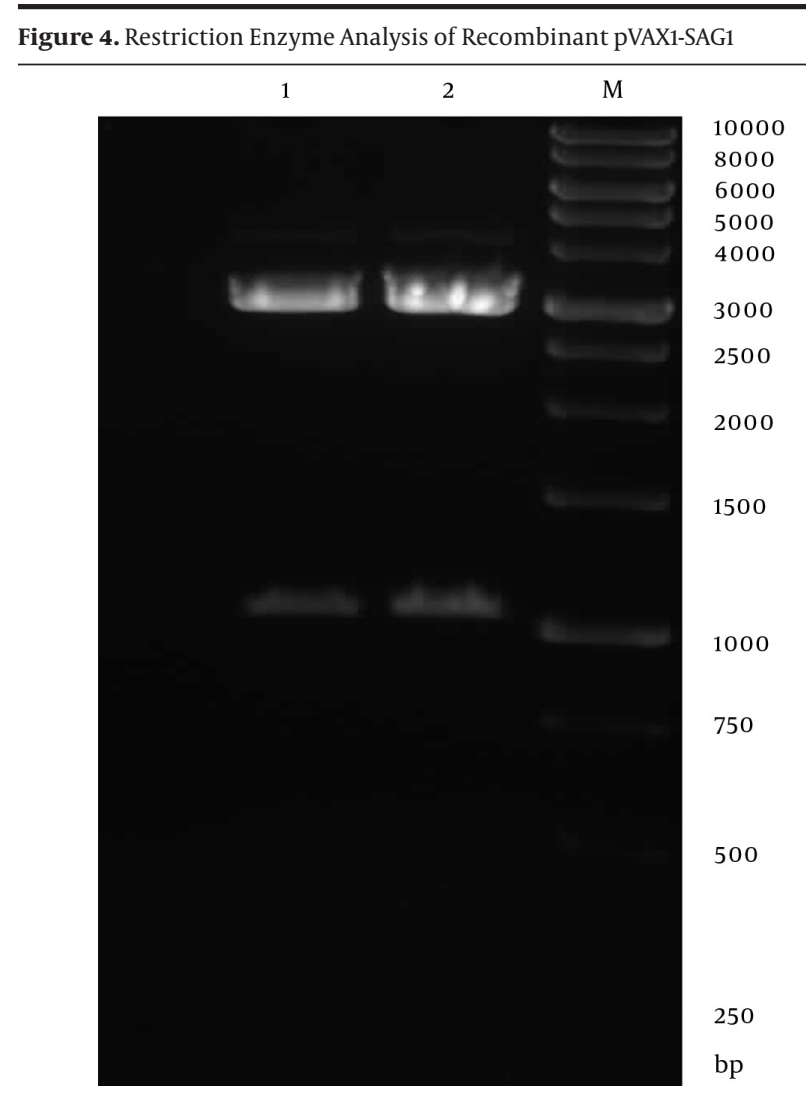

Line 1 and 2, the recombinant pVAX-SAG1 plasmid digested by NheI and XhoI; Line M, 1 kb DNA ladder. 


\begin{tabular}{|c|c|c|c|}
\hline Query & 1 & MSVSLHHFIISSGFLTSMFPKAVRRAVTAGVFAAPTLMSFLLCGVMASDPPLVANQVVTC & 80 \\
\hline & & MSVSLHHFIISSGFLTSMFPKAVRRAVTAGVFAAPTLMSFL CGVMASDPPLVANQVVTC & \\
\hline sbjct & 1 & MSVSLHHFIISSGFLTSMFPKAVRRAVTAGVFAAPTLMSFLRCGVMASDPPLVANQVVTC & 60 \\
\hline Query & 181 & PDKKSTAAVILTPTENHFTLKCPKTALTEPPTLAYSPNRQICPAGTTSSCTSKAVTLSSL & \\
\hline Sbjet & 61 & PDKKSTAAVILTPTENHFTLKCPKTALTEPPTLAYS PNRQICPAGTTSSCTSKAVTLSSL & \\
\hline Query & 361 & IPEAEDSWWTGDSASLDTAGIKLTVPIEKFPVTTQTFVVGCIKGDDAQSCMVTVTVQARA & \\
\hline Sbjet & 121 & IPEAEDSWWTGDSASLDTAGIKITVPIEKFPVTTQTFVVGCIKGDDAQSCMVTVTVQARA & \\
\hline Query & 541 & $\begin{array}{l}\text { SSVVNNVARCSYGADSTLGPVKLSAEGPTTMTLVCGKDGVKVPQDNNQYCSGTTLTGCNE } \\
\text { SSVVNNVARCSYGADSTLGPVKLSAEGPTTMTLVCGKDGVKVPQDNNQYCSGTTLTGCNE }\end{array}$ & \\
\hline Sbjet & 181 & SSVVNNVARCSYGADSTLGPVKLSAEGPTTMTLVCGKDGVKVPQDNNQYCSGTTLTGCNE & \\
\hline Query & 721 & $\begin{array}{l}\text { KSFKDILPKLTENPWQGNASSDKGATLTIKKEAFPAESKSVI IGCTGGS PEKHHCTVKLE } \\
\text { KSFKDILPKLTENPWQGNASSDKGATLTIKKEAFPAESKSVI IGCTGGS PEKHHCTVKLE }\end{array}$ & \\
\hline Sbjct & 241 & KSFKDILPKLTENPWQGNASSDKGATLTIKKEAFPAESKSVIIGCTGGS PEKHHCTVKLE & \\
\hline Query & 901 & $\begin{array}{l}\text { FagaagsaesaagtasHVSIFAMVIGLIGSI } 993 \\
\text { FAGAAGS +SAAGTASHVSIFAMVIGLIGSI }\end{array}$ & \\
\hline Sbjet & 301 & FAGAAGSEKSAAGTASHVSIFAMVIGLIGSI 331 & \\
\hline
\end{tabular}

Figure 5. Alignment of Amino Acids Sequence of T. gondii SAG1 Fragment Compared with Amino Acid Sequence of SAG1 Accession Number AFO54881.1, AFO54884.1, AFO54882.1 and ACT64636.1 Respectively

Among antigenic components of T. gondii, SAG1 is a major surface antigen, which was identified on the surface membrane of this protozoa using a monoclonal antibody by Handman et al. in 1980 (30). This protein is a crucial ligand for attachment and promoting invasion of tachyzoites to host cells and is able to induce both effective and durable humoral and cellular immune responses. Thus, SAG1 is a protein with an excellent antigenicity and immunogenicity to develop effective diagnosis tests or vaccines $(23,31)$. Additionally, among different strains of $T$. gondii, the sequence of SAG1 is highly conserved and share a high degree of homology between type I (pathogenic and lethal to mice) and type II/III (cystogenic) strains (32). Therefore, in recent years many researchers have tried to express SAG1 in different hosts including $E$. coli, Pichia pastoris, baculovirus and insect cell in order to use recombinant protein and DNA vaccine of SAG1 in experimental animals and or use it in diagnosis in serological methods $(18,19)$.

The recombinant SAG1 produced in E. coli often loses its specific immunogenicity; therefore, it was necessary to refold this protein or use the truncated SAG1 in serological methods (33). Kazemi et al. cloned a $957 \mathrm{bp}$ fragment of SAG1 of RH T. gondii and studied its expression in E. coli to use this antigen for diagnosis of chronic and acute toxoplasmosis using using Enzyme Linked Immunosorbant Assay (ELISA) (34).

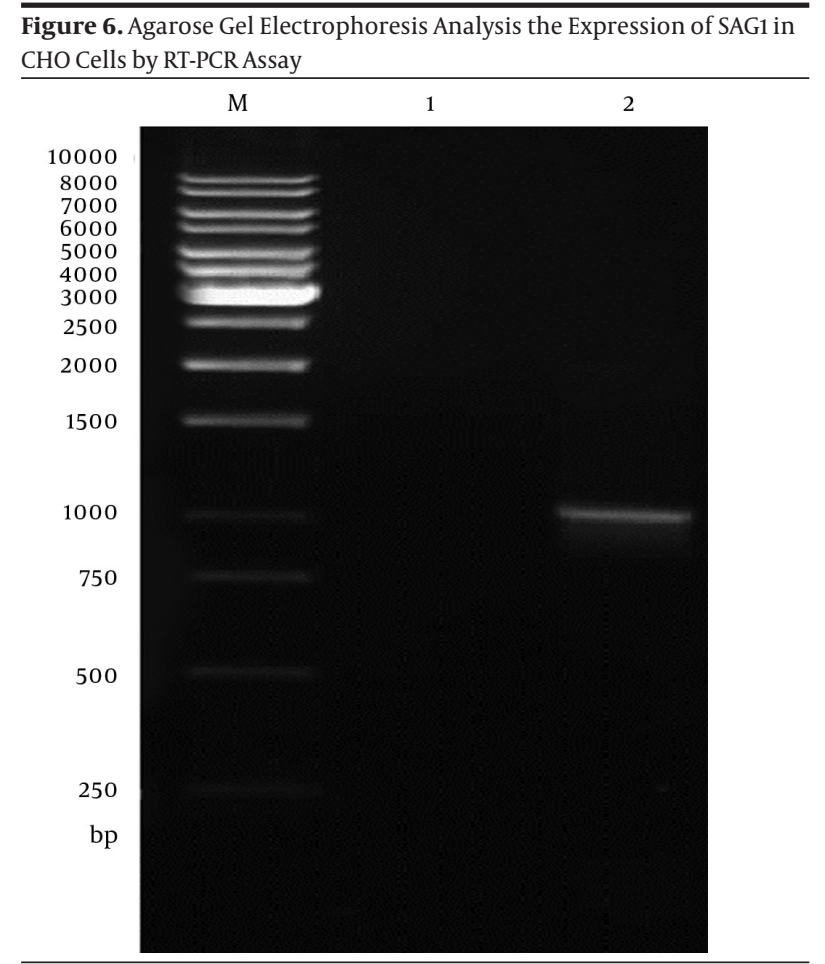

Line M, 1 kb DNA ladder; Line 1, Transfected CHO cells with empty pVAX1 plasmid; Line 2, Transfected CHO cells with pVAX-SAG1. 
Figure 7. Expression Analysis of SAG1 in CHO Cells by Immunofluorescence Assay

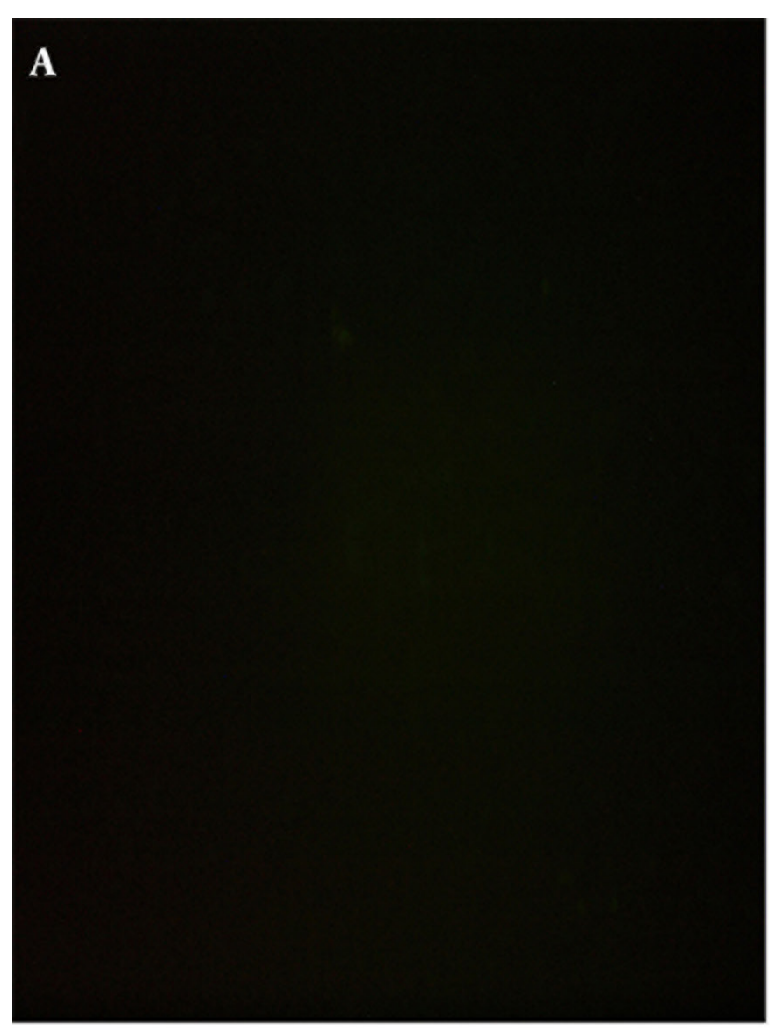

B
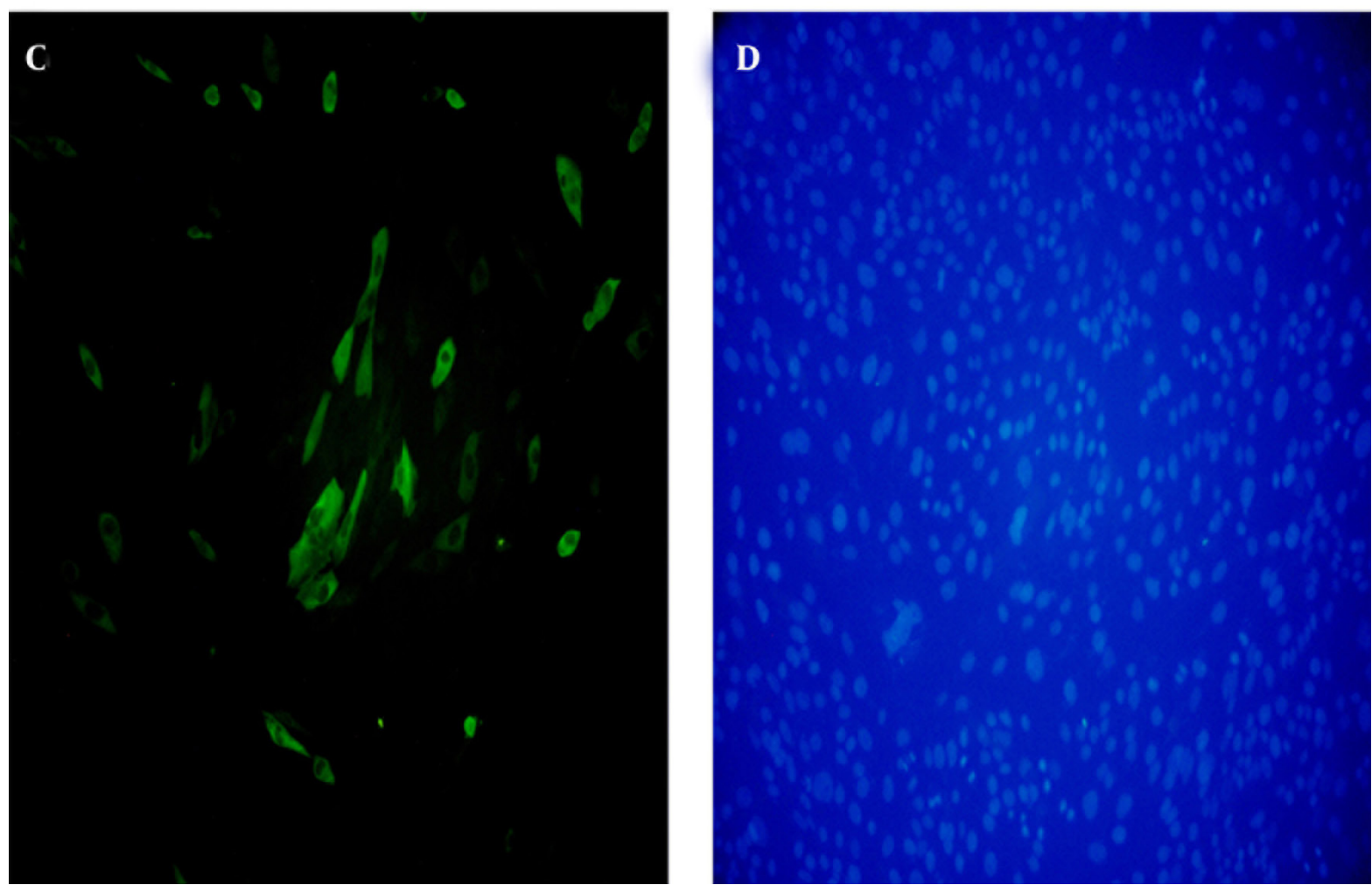

A) Transfected CHO cells with empty pVAX1 plasmid; B) DAPI staining of negative control group; C) Transfected CHO cells with pVAX1-SAG1 and revealed using anti-SAG1 monoclonal antibody and secondary antibody labeled with FITC; D) DAPI was used to stain the cell nuclei. 
Figure 8. Expression of SAG1 Protein in Transfected CHO Cell Lysate by Western Blot

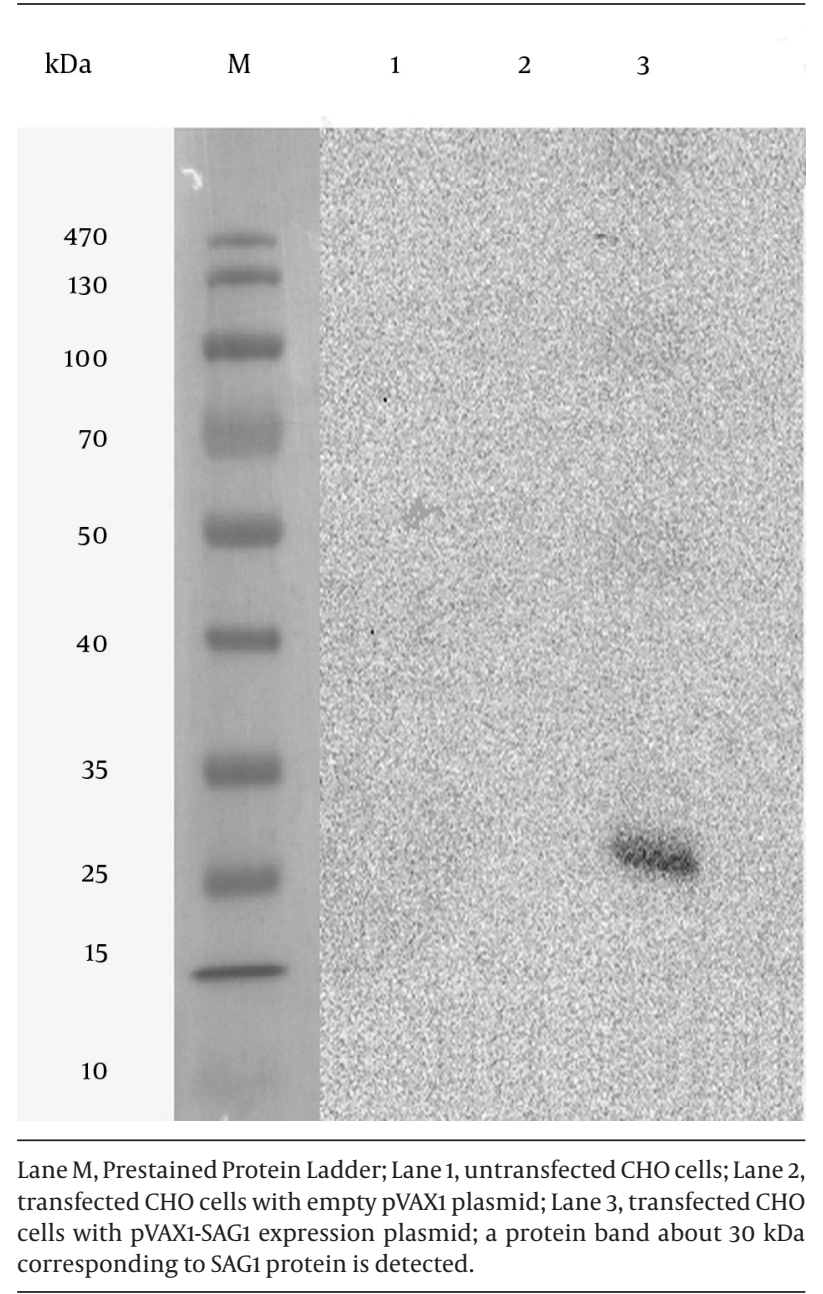

Khanaliha et al. used this antigen in combination of SAG2 and SAG3 for serodiagnosis of toxoplasmosis in human (35). We cloned a 1020 bp of SAG1 of T. gondii in the pVAX vector and transfect CHO cells. In other study, Solhjoo et al. cloned a $960 \mathrm{bp}$ fragment of SAG1 of T. gondii into a pTZ57R cloning vector then transformed it in a E.coli TG1 strain (36). Makioka et al. cloned SAG1 gene of T. gondii into several vectors, including pTV118N, pKK233-2 and pGEX-1 then transferred it to several E. coli strains (JM109, JM105, etc.) to investigate the expression of this protein. Their results indicate that among these combinations, the pGEX-1 recombinant plasmid achieved a high expression level using a Glutathione S-transferase (GST)-fusion protein and this protein could be able to activate macrophages in animal experiments and to exhibit toxoplasmacidal effects in vitro (37).

Nielsen et al. was cloned SAG1 gene of T. gondii in frame with a sequence encoding a synthetic mimic of the tPA signal into a eukaryotic expression vector and evaluated immunization of this plasmid in $\mathrm{C} 3 \mathrm{H}(\mathrm{H}-2 \mathrm{k})$ and $\mathrm{BALB} / \mathrm{C}$ (H-2D) mice (38). In another survey, Solhjoo et al. (36) cloned a 960 bp fragment of T. gondii SAG1 gene into a pTZ57R/T plasmid and then transferred it to pcDNA3 to evaluate its immunization with aluminum phosphate as an adjuvant in BALB/c mice. Hoseinian Khosroshahi et al. used this plasmid (pcDNA3-SAG1) and pcDNA3-ROP2 in a DNA vaccine cocktail approach to evaluate the immune response against toxoplasmosis (39). In another study, Chuang et al. investigated protective immunity of recombinant SAG1 protein into the plasmid pGEX-6P-1 and expressed as a GST fusion protein in BL21 (DE3) E. coli and then, rSAG1 was encapsulated into PLG (poly lactideco-glycolide) microparticles using the double emulsion method and intraperitoneally injected into BALB/c mice (40). Qu et al. cloned the SAG1 gene into pcDNA3 and transformed into Salmonella typhimurium competent cells then assessed the immune responses of female ICR mice vaccinated by oral inoculation (32). Also, Meng et al. cloned the SAG1 gene and 14-3-3 gene of T. gondii into eukaryotic expression pBudCE4.1 then evaluated the protective immune responses of them in BALB/c mice (41).

$\mathrm{pVAX1}^{\mathrm{TM}}$ is a $3.0 \mathrm{~kb}$ plasmid vector that was constructed by modifying pcDNA ${ }^{\mathrm{TM}} 3.1$ for use in the development of DNA vaccines. The vector was constructed to be consistent with the Food and Drug Administration (FDA) regulations. The vector was constructed to be consistent with the Food and Drug Administration (FDA) regulations. This vector allow high-copy number replication in E. coli and contains a human cytomegalovirus (CMV) immediate-early promoter for high-level expression in a wide range of mammalian cells, Bovine Growth Hormone (BGH), poly-adenylation signal for efficient transcription termination and poly-adenylation of mRNA and kanamycin resistance gene for selection in E. coli. Therefore, recently many investigators became interested to use the pVAX1 vector plasmid for DNA vaccine experimental designs. Hence, some antigens of T. gondii, including SAG1, ROP2, GRA4 and MIC3 have cloned into the pVAX1 plasmid and used for DNA vaccine (19).

Wu et al. constructed a multicomponent DNA vaccine by encoding GRA1 and SAG1 genes of T. gondii in the PVAX1 vector and evaluated the protective ability of this construction against toxoplasmosis in BALB/c mice, which were challenged with tachyzoites (42). Zhou et al. also cloned the SAG1 and GRA2 from T. gondii plus surface antigen of hepatitis $B$ virus in the pVAX vector as a novel genetic adjuvant to evaluate immunization of those DNA plasmids against toxoplasmosis in BALB/C mice (43). In current study, the DNA sequence encoding fragment of T. gondii (RH) SAG1 was amplified by specific primer, including Kozak sequence and cloned into pVAX1 expression vector under a CMV promoter. The Kozak consensus sequence, GCCACCATGT, was introduced in $5^{\prime}$ of the SAG1 DNA, which was demonstrated useful earlier for translational control and optimization of expression vectors in an eukaryotic organism (44).

Sequence analysis of SAG1 cloned into the pVAX1 shows that the sequence has $100 \%$ identity with TgCatBr1strain, 
FOU strain, GUY-1992-RUB strain and high homology of 99\% with RH strain. Then, the expression of this gene was confirmed by RT-PCR, indirect immunofluorescence test and western blot analysis. In this study, unlike other surveys, the aimed to express full length of SAG1 in the pVAX1 expression vector to evaluate its immunity with DNA vaccine in future investigations. These results confirmed that the recombinant plasmid was successfully constructed and expressed in vitro and recombinant $\mathrm{Tg}$ SAG1 protein possessed immunological activity that can be used for DNA vaccine in further investigations.

\section{Acknowledgements}

The authors would like to thank the staff of the cell and molecular research center and the department of medical parasitology, school of medicine and vice chancellor of research development and technology, Ahvaz Jundishapur University of Medical Sciences, Ahvaz, Iran. All authors have read and approved the final manuscript.

\section{Authors' Contributions}

This paper is extracted from Dr. Rahman Abdizadeh thesis (D/708). Sharif Maraghi and Ata A. Ghadiri were supervisors and Mehdi Tavalla and Saeedeh Shojaee were advisors of the thesis. Sharif Maraghi and Ata A. Ghadiri take responsibility for the accuracy of the data. Ata A. Ghadiri and Rahman Abdizadeh contributed equally to the design and performance of the study. Ata A. Ghadiri, Mehdi Tavalla and Saeedeh Shojaee participated in the laboratory evaluation. Sharif Maraghi and Ata A. Ghadiri performed the literature review, drafted the manuscript and are the guarantors.

\section{Financial Disclosure}

This study was a part of Dr. Rahman Abdizadeh, Ph.D thesis of medical parasitology, financially supported by Ahvaz Jundishapur University of Medical Sciences, Ahvaz, Iran.

\section{Funding/Support}

The study was supported financially by the grant (No. CMRC-71) provided by cell and molecular research center, School of medicine, deputy of research, Ahvaz Jundishapur University of Medical Sciences, Ahvaz, IR Iran.

\section{References}

1. Weiss LM, Kim K. Toxoplasma gondii : the model apicomplexan, perspectives and methods. 2 edLondon: Academic Press; 2014.

2. Tenter AM, Heckeroth AR, Weiss LM. Toxoplasma gondii: from animals to humans. Int J Parasitol. 2000;30(12-13):1217-58.

3. Dubey JP. History of the discovery of the life cycle of Toxoplasma gondii. Int J Parasitol. 2009;39(8):877-82.

4. Elmore SA, Jones JL, Conrad PA, Patton S, Lindsay DS, Dubey JP. Toxoplasma gondii: epidemiology, feline clinical aspects, and prevention. Trends Parasitol. 2010;26(4):190-6.

5. Jones JL, Dubey JP. Foodborne toxoplasmosis. Clin Infect Dis. 2012;55(6):845-51.
6. Izadi M, Jonaidi Jafari N, Mahmoodzadeh Poornaki A, Sadraei J, Rezavand B, Mirzaei HR, et al. Detection of Toxoplasma gondii from Clinical Specimens of Patients Receiving Renal Transplant Using ELISA and PCR. Nephrourol Mon. 2013;5(5):983-7.

7. Sarkari B, Shafiei R, Zare M, Sohrabpour S, Kasraian L. Seroprevalence and molecular diagnosis of Toxoplasma gondii infection among blood donors in southern Iran. J Infect Dev Ctries. 2014;8(4):543-7.

8. Chiang TY, Kuo MC, Chen CH, Yang JY, Kao CF, Ji DD, et al. Risk factors for acute Toxoplasma gondii diseases in Taiwan: a populationbased case-control study. PLoS One. 2014;9(3).

9. Torgerson PR, Mastroiacovo P. The global burden of congenital toxoplasmosis: a systematic review. Bull World Health Organ. 2013;91(7):501-8.

10. Daryani A, Sarvi S, Aarabi M, Mizani A, Ahmadpour E, Shokri A, et al. Seroprevalence of Toxoplasma gondii in the Iranian general population: a systematic review and meta-analysis. Acta Trop. 2014;137:185-94.

11. Munoz M, Liesenfeld O, Heimesaat MM. Immunology of Toxoplasma gondii. Immunol Rev. 2011;240(1):269-85.

12. Pereira-Chioccola VL, Vidal JE, Su C. Toxoplasma gondii infection and cerebral toxoplasmosis in HIV-infected patients. Future Microbiol. 2009;4(10):1363-79.

13. Yan J, Huang B, Liu G, Wu B, Huang S, Zheng H, et al. Meta-analysis of prevention and treatment of toxoplasmic encephalitis in HIVinfected patients. Acta Trop. 2013;127(3):236-44

14. Saadatnia G, Golkar M. A review on human toxoplasmosis. Scand J Infect Dis. 2012;44(11):805-14.

15. Paquet C, Yudin MH, Society of O, Gynaecologists of C. Toxoplasmosis in pregnancy: prevention, screening, and treatment. $\mathrm{J} \mathrm{Ob}$ stet Gynaecol Can. 2013;35(1):78-81.

16. Chen J, Zhou DH, Li ZY, Petersen E, Huang SY, Song HQ, et al. Toxoplasma gondii: protective immunity induced by rhoptry protein 9 (TgROP9) against acute toxoplasmosis. Exp Parasitol. 2014;139:42-8.

17. Meng M, Zhou A, Lu G, Wang L, Zhao G, Han Y, et al. DNA prime and peptide boost immunization protocol encoding the Toxoplasma gondii GRA4 induces strong protective immunity in BALB/c mice. BMC Infect Dis. 2013;13:494.

18. Zhang NZ, Chen J, Wang M, Petersen E, Zhu XQ. Vaccines against Toxoplasma gondii: new developments and perspectives. Expert Rev Vaccines. 2013;12(11):1287-99.

19. Garcia JL, Innes EA, Katzer F. Current progress toward vaccines against Toxoplasma gondii. Vaccine Dev Therap. 2014;4(1):23-8.

20. Couvreur G, Sadak A, Fortier B, Dubremetz JF. Surface antigens of Toxoplasma gondii. Parasitology.1988;97 ( Pt 1):1-10.

21. Grimwood J, Smith JE. Toxoplasma gondii: the role of parasite surface and secreted proteins in host cell invasion. Int J Parasitol. 1996;26(2):169-73.

22. Tomavo S, Schwarz RT, Dubremetz JF. Evidence for glycosyl-phosphatidylinositol anchoring of Toxoplasma gondii major surface antigens. Mol Cell Biol.1989;9(10):4576-80.

23. Wang Y, Yin H. Research progress on surface antigen 1(SAG1) of Toxoplasma gondii. Parasit Vectors. 2014;7:180.

24. Sudan V, Tewari AK, Singh H. An Insight into the Behavior, Course and Kinetics of Acute Infection of Toxoplasma gondii Human $\mathrm{RH}$ Strain in Experimentally Infected Murine Model. Iran J Parasitol. 2014;9(1):114-9.

25. Cohen SN. Preparation and transformation of compete E. coli using calcium chloride.New York: Cold Spring Harbor Laboratory Press; 2001.

26. Shevach EM. London: John Wiley and Sons, Inc.; 2002.

27. Laemmli UK. Cleavage of structural proteins during the assembly of the head of bacteriophage T4. Nature.1970;227(5259):680-5.

28. Towbin H, Staehelin T, Gordon J. Electrophoretic transfer of proteins from polyacrylamide gels to nitrocellulose sheets: procedure and some applications. Proc Natl Acad Sci U S A. 1979;76(9):4350-4.

29. Okuda K, Wada Y, Shimada M. Recent Developments in Preclinical DNA Vaccination. Vaccines. 2014;2(1):89-106.

30. Handman E, Goding JW, Remington JS. Detection and characterization of membrane antigens of Toxoplasma gondii. J Immunol. 
1980;124(6):2578-83.

31. Mineo JR, Kasper LH. Attachment of Toxoplasma gondii to host cells involves major surface protein, SAG-1 (P30). Exp Parasitol. 1994;79(1):11-20.

32. Qu D, Wang S, Cai W, Du A. Protective effect of a DNA vaccine delivered in attenuated Salmonella typhimurium against Toxoplasma gondii infection in mice. Vaccine. 2008;26(35):4541-8.

33. Chen XG, Gong Y, Hua L, Lun ZR, Fung MC. High-level expression and purification of immunogenic recombinant SAG1 (P30) of Toxoplasma gondii in Escherichia coli. Protein Expr Purif. 2001;23(1):33-7.

34. Kazemi B, Bandehpour M, Maghen L, Solgi G. Gene Cloning of 30 kDa Toxoplasma gondii Tachyzoites Surface Antigen (SAG1). Iran J Parasitol. 2007;2(2):1-8.

35. Khanaliha K, Motazedian MH, Kazemi B, Shahriari B, Bandehpour M, Sharifniya Z. Evaluation of recombinant SAG1, SAG2, and SAG3 antigens for serodiagnosis of toxoplasmosis. Korean J Parasitol. 2014;52(2):137-42.

36. Solhjoo K, Ghaffari F, Dalimi-Asl A, Sharifi Z. Enhancement of Antibody Immune Response to a Toxoplasma gondii SAG1-Encoded DNA Vaccine by Formulation with Aluminum Phosphate. J Med Sci. 2007;7(3):361-7.

37. Makioka A, Kobayashi A. Toxoplasmacidal activity of macrophages activated by recombinant major surface antigen (P30) of Toxoplasma gondii. Infect Immun. 1991;59(8):2851-2.

38. Nielsen HV, Lauemoller SL, Christiansen L, Buus S, Fomsgaard A,
Petersen E. Complete protection against lethal Toxoplasma gondii infection in mice immunized with a plasmid encoding the SAG1 gene. Infect Immun. 1999;67(12):6358-63.

39. Hoseinian Khosroshahi K, Ghaffarifar F, D'Souza S, Sharifi Z, Dalimi A. Evaluation of the immune response induced by DNA vaccine cocktail expressing complete SAG1 and ROP2 genes against toxoplasmosis. Vaccine. 2011;29(4):778-83.

40. Chuang SC, Ko JC, Chen CP, Du JT, Yang CD. Induction of longlasting protective immunity against Toxoplasma gondii in BALB/c mice by recombinant surface antigen 1 protein encapsulated in poly (lactide-co-glycolide) microparticles. Parasit Vectors. 2013;6:34.

41. Meng M, He S, Zhao G, Bai Y, Zhou H, Cong H, et al. Evaluation of protective immune responses induced by DNA vaccines encoding Toxoplasma gondii surface antigen 1 (SAG1) and 14-3-3 protein in BALB/c mice. Parasit Vectors. 2012;5:273.

42. Wu XN, Lin J, Lin X, Chen J, Chen ZL, Lin JY. Multicomponent DNA vaccine-encoding Toxoplasma gondii GRA1 and SAG1 primes: anti-Toxoplasma immune response in mice. Parasitol Res 2012;111(5):2001-9.

43. Zhou H, Min J, Zhao Q, Gu Q, Cong H, Li Y, et al. Protective im mune response against Toxoplasma gondii elicited by a recombinant DNA vaccine with a novel genetic adjuvant. Vaccine. 2012;30(10):1800-6.

44. Kozak M. Regulation of translation via mRNA structure in prokaryotes and eukaryotes. Gene. 2005;361:13-37. 